\title{
Lamivudine today: Experience in a single Bulgarian center
}

\author{
Donika Krasteva $^{1}$, Deian Jelev ${ }^{1}$, Krassimir Antonov ${ }^{1}$, \\ Zoya Spassova ${ }^{1}$, Rossen Nikolov ${ }^{1}$, Georgi Mihailov ${ }^{2}$, \\ Branimir Spassov ${ }^{2}$, Rasho Rashkov ${ }^{3}$, Zlatimir \\ Kolarov $^{3}$, Lyudmila Mateva ${ }^{1}$, Zahariy Krastev ${ }^{1}$
}

1. Clinic of Gastroenterology, St. Ivan Rilsky University Hospital, Sofia Medical University;

2. National Specialized Hospital for Active Therapy of Hematological Diseases, Sofia Medical University;

3. Clinic of Rheumatology, St. Ivan Rilsky University Hospital, Sofia Medical University.

\begin{abstract}
Lamivudine (LAM) is not recommended in the treatment of chronic hepatitis $B$ due to high-rate of resistance: $49 \%$ to $70 \%$ after 3rd and 5th treatment year. LAM-resistance is more frequent in cases with high viral load or lack of complete HBV-DNA suppression within 6 months. Antiviral effect of LAM is rapid and potent if HBV-DNA level is low. Subjects who are indicated for prophylactic NUC-therapy prior immunosuppression as well as cirrhotics are with relatively low viral load, but data on the LAM efficacy in such patient populations are limited.
\end{abstract}

We evaluated 97 patients (56 males) with chronic HBV-infection, mean age 56.5 ( \pm 13.1 ) years. Of them: 54 (56\%) were with liver cirrhosis; 18 (18.5\%) were with hematological malignancy or carcinoma and 16 (16.5\%) with systemic autoimmune diseases. The rest 9 patients were of older age or started LAM after unsuccessful IFN-therapy. Initially 88 (91\%) were HBeAg-negative. Co-infections with HCV and HDV were excluded. Patients received LAM 100mg/d for at least 36 months or until development of resistance. HBV-DNA was measured on 3-month interval by real-time PCR. Test for LAM-resistant mutations was performed in case of viral breakthrough. 
At the treatment-month 6; 12; 24 and 36 a complete viral suppression was achieved in 73\%; 89\%; 95\% and $96 \%$ of patients, respectively. During therapy 16/97(16.6\%) developed LAM-resistance for median 18 (9-36) months.

Resistant patients were with higher median HBV-DNA: 28750000 (11751 - 7570000000) cp/mL vs. 949300 (380 - 4650000000) cp/mL; $p<0.05$. The complete viral suppression was induced more slowly in patients who developed drug resistance: median 9 (3-36) months vs. 3 (1 - 27); p<0.001. LAMtherapy was safe and very well tolerated. All patients with LAM-resistant mutations were switched to tenofovir and HBV-DNA rapidly decreased, to undetectable level within 6 months. Rescue therapy lead to durable and complete viral suppression. ALT-flare or hepatic decompensation was not observed.

In conclusion, LAM is effective, safe and cost-saving treatment option in selected patients with low baseline viral load. In this case LAM induces a rapid and durable viral suppression with risk of resistance far below previously reported levels. Patients should be carefully monitored during therapy. Tenofovir must be started immediately after viral breakthrough to avoid ALT flare and potential hepatic decompensation.

\section{Keywords: lamivudine resistance, liver cirrhosis, immunosuppression, hematological malignances, systemic autoimmune diseases.}

\section{Introduction}

Despite the significant advances in the antiviral therapies and vaccination programs, hepatitis $B$ virus (HBV) infection remains a major health problem. It has affected about two billion people worldwide, causing chronic hepatitis in up to 240 million individuals (1). In Bulgaria, HBV is with intermediate prevalence of 3.9\%.(2,3) Similarly to other South European and Mediterranean countries, about $90 \%$ of Bulgarian patients with chronic hepatitis $B(\mathrm{CHB})$ are hepatitis $B$ "e" antigen ( $\mathrm{HBeAg}$ )-negative and genotype $D$ was found in almost $100 \%$ of them. $(4,5)$

Treatment strategies for $\mathrm{CHB}$ include achieving a sustained immune control by a finite treatment course with pegylated interferon (Peg IFN), or maintained on-treatment viral control by suppression of HBV replication with nucleoside/nucleotide analogues (NUCs). NUCs are the only treatment option for patients with advanced cirrhosis as well as for subjects with autoimmune diseases and those on immunosuppressive therapy (6).

Lamivudine (LAM) was the first NUC introduced in the clinical practice, but currently it is not recommended in the treatment of $\mathrm{CHB}$ due to the high rate of drug resistance, which was found to be $24 \%$ after the 1st treatment year and increases to $49 \%$ and $70 \%$ after 3 and 5 years of LAM therapy, respectively (6). Resistance occurs more frequently in subjects with high viral load and in those who failed to achieve complete HBV DNA suppression within 6 months $(7,8)$.

Theoretically, antiviral effect of LAM should be rapid and potent in patients with low HBV DNA level. The majority of subjects that are indicated for prophylactic NUC therapy prior initiation of immunosuppressive treatment as well as cirrhotics are with relatively low viral load, but data on the LAM efficacy and resistance in such patient populations are limited. 


\section{Material and methods}

\section{Patients}

Ninety-seven patients with chronic HBV infection and with mean age 56.5 ( \pm 13.1$)$ years were evaluated. They were 56 males (mean age $55.9 \pm 14$ years) and 41 females (mean age $57.4 \pm 11.6$ years). Of total 97 subjects: 54 (56\%) were with liver cirrhosis; 18 (18.5\%) were with hematological malignancy or carcinoma and $16(16.5 \%)$ with systemic autoimmune diseases. The rest 9 patients were of older age and with significant co-morbidities or started LAM due to hepatic flare after unsuccessful IFN therapy. All subjects were viremic with presumed HBV genotype D as previous studies in Bulgarian patients found $100 \%$ genotype D (4, 5). The median baseline viral load was $1151213(380-7570000$ 000) cp/mL. Initially 88 (91\%) were HBeAg-negative. Co-infections with HCV, HDV and HIV were excluded.

\section{Treatment}

Patients were treated with $100 \mathrm{mg}$ lamivudine daily (ZeffixTM, GlaxoSmithKline) for at least 36 months or until development of drug resistance. During therapy subjects were evaluated on 3-month interval clinically and by testing of hematology, blood chemistry, liver enzymes and HBV viral load.

In case of viral breakthrough the treatment compliance was checked and test for HBV-mutations associated with LAM resistance was performed. Patients who developed LAM resistance were switched to tenofovir (VireadTM, Gilead) and were carefully evaluated until serum HBV DNA decreases to undetectable level.

\section{Measurements}

Serum HBV DNA levels were measured by using LightCycler real time polymerase chain reaction assay (PCR) (Roche Diagnostics).

HBV mutations associated with LAM resistance were detected by a line probe assay (INNO-LiPA DR, Innogenetics). Serological testing for HBeAg/anti-HBe, anti-HDV, anti-HCV and anti-HIV were performed by commercially available ELISA kits. Routine laboratory methods were used for testing of hematology and blood chemistry parameters.

\section{Results}

During therapy HBV DNA decreased to undetectable serum level in 93/97 (96\%) of patients. The median time of achieving undetectable serum HBV DNA was $6(1-36)$ months after LAM initiation. At the treatment month $6 ; 12 ; 24$ and 36 a complete viral suppression was achieved in 73\%; 89\%; 95\% and 96\% of patients, respectively. As expected a strong positive correlation was found between the baseline viral load and the timeframe of achieving undetectable serum HBV DNA $(r=0.6 ; p<0.05)$. Only 4/97 (4\%) of patients remained viremic during LAM therapy. All of them were with extremely high baseline viral load (> $1000000000 \mathrm{cp} / \mathrm{mL})$.

During therapy 16/97 of patients (16.6\%) developed resistance to LAM, which occurred median 18 (9-36) months after treatment initiation. Patient with drug resistance compared to those without resistance were 
with higher median baseline HBV DNA: 28750000 (11 751-7 570000 000) cp/mL vs. 949300 (380-4 $650000000) \mathrm{cp} / \mathrm{mL} ; \mathrm{p}<0.05$. The rate of LAM resistance progressively increases with respect to baseline viral load. Resistance were not observed among patients with low viral load $(<10000 \mathrm{cp} / \mathrm{mL})$, while the rate of LAM resistance was about 10\% in subjects with baseline HBV DNA between 10000 and $1000000 \mathrm{cp} / \mathrm{mL}$ and increased to $27 \%$ in those with very high viral load (> $100000000 \mathrm{cp} / \mathrm{mL}$ ) - figure 1 .

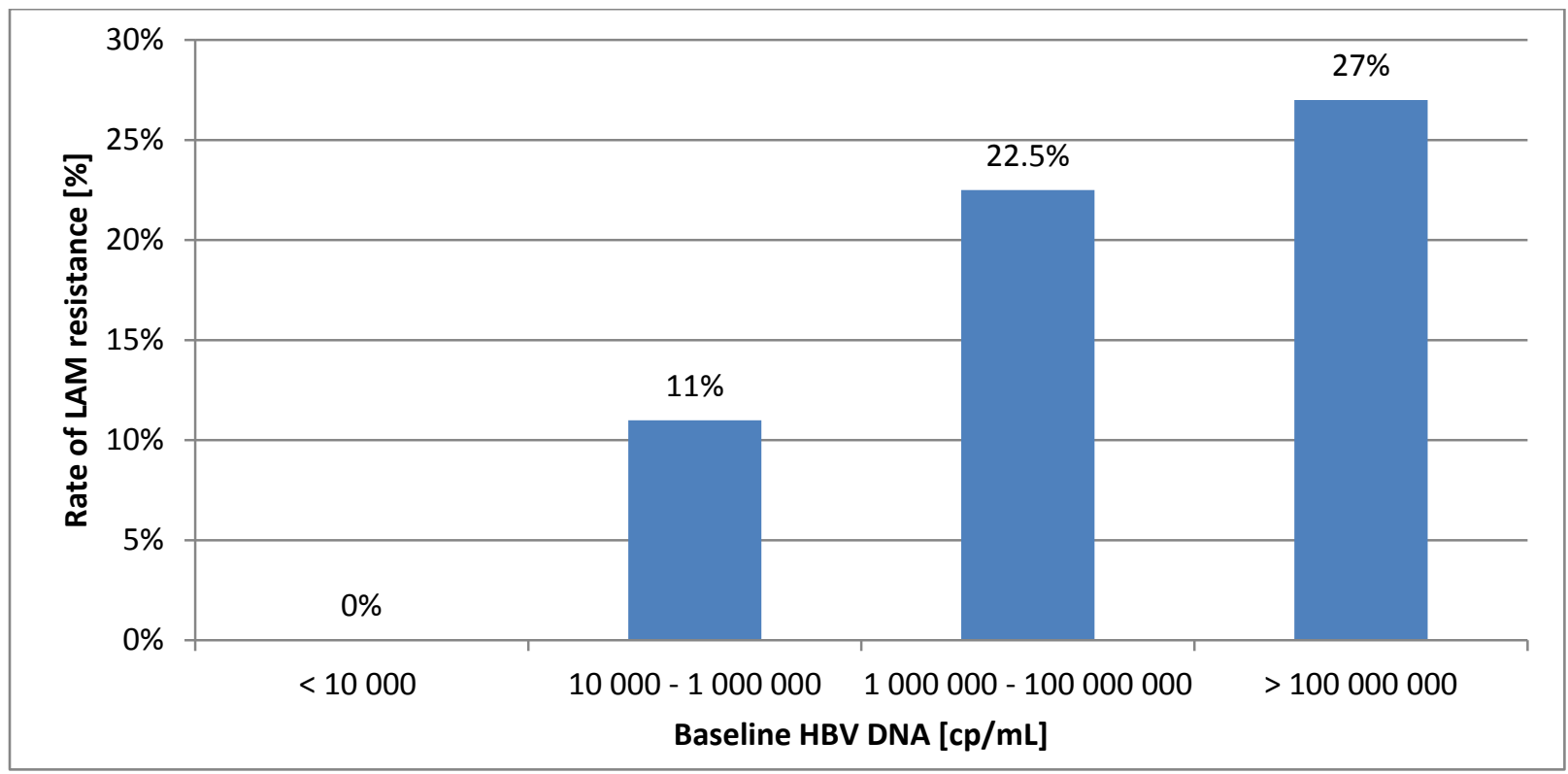

Figure 1. Rate of LAM resistance according to the baseline viral load

In addition, the viral suppression was induced more slowly in patients who developed drug resistance compared to those without resistance. The median time of achieving undetectable HBV DNA in patients who developed LAM resistance was 9 (3 - 36) months vs. 3 (1-27) months in non-resistant subjects; $p<$ 0.001 .

LAM therapy was safe and very well tolerated. In all subjects with evidence of drug resistance HBVmutations were detected during the viral breakthrough, but prior to the expected ALT elevation. Patients were switched to tenofovir and HBV DNA rapidly decreased, to undetectable level within 6 months. Rescue therapy lead to sustained and complete viral suppression. ALT flare or hepatic decompensation was not observed.

\section{Discussion}

Our results clearly demonstrate that in the selected subjects with low baseline viral load LAM induces a rapid and durable viral suppuration with relatively low resistance rate of only $16.6 \%$ after 36-month therapy which is about 3 times lower than previously reported $49 \%$ for the same treatment duration (6). Our data 
are fully in line with findings of recent study from China that reported similar resistance rate of $15.6 \%$ after 2 years of LAM therapy among 256 patients with decompensated HBV cirrhosis (9).

Resistance is the most important limitation of LAM as it may lead to ALT flares and severe hepatic decompensation (10). The risk of liver failure is higher in the cirrhotic patients due to the limited functional hepatic reserve associated with advanced liver disease (11). It is well known that flares occur after viral breakthrough, so they can be avoided by careful monitoring of HBV DNA and rapid implementation of rescue therapy with tenofovir (11). By such an approach we did not observe ALT flare or hepatic decompensation among cases with LAM resistance.

Entecavir and tenofovir have been recommended for the first-line treatment options in CHB, but due to the high cost they are not used widespread in the countries with limited resources. On the other hand LAM is still widely used in the economically less developed regions (9). It was confirmed that LAM is highly costeffective in patients with cirrhosis (9). Although generic tenofovir is already available in the market its price remains still about 3 times higher than LAM, so LAM will be still used in many countries with limited health resources.

\section{Conclusion}

LAM may be effective, safe and cost-saving treatment option in selected patients with low baseline viral load, as in this setting it induces a rapid and durable viral suppression with risk of resistance far below previously reported levels. Patients should be carefully monitored during therapy. Drug resistance should be suspected in subjects with high baseline viremia or who fail to achieve complete and rapid viral suppression within the first 6 months. Rescue therapy with tenofovir must be started immediately after detection of viral breakthrough and drug resistance mutilations to avoid ALT flare and potential hepatic decompensation.

\section{References}

1. Ott JJ, Stevens GA, Groeger J, Wiersma ST. Global epidemiology of hepatitis B virus infection: new estimates of age-specific HBsAg seroprevalence and endemicity. Vaccine. 2012; 30:221219.

2. Petrunov B, Kojouharova $M$, Teoharov $P$ et al. Seroepidemiology study on Hepatitis $C$ and $B$ viral infections prevalence in Bulgaria and Northern Greece. J Hepatol 2002; 36: 138-139.

3. Kevorkyan A, Teoharov P, Lernout T et al. Prevalence of HBV and HCV among outpatients in the Plovdiv region of Bulgaria. J Med Virol. 2015;87(3): 401-406.

4. Krastev Z, Tchernev K, Petrova D et al. An open label, multicenter, national non-randomized study to evaluate efficacy and safety of Peginterferon alfa-2a (40 KD) in patients with HBeAgnegative chronic hepatitis B: preliminary results. Bulgarian Hepatogastroenterol 2006; 2: 90-91.

5. Jelev D. Chronic HBV infection: characteristics, natural course and options for treatment DMSc thesis, Sofia 2010.

6. European association for the study of the liver (EASL). EASL 2017 Clinical Practice Guidelines on the management of hepatitis B virus infection. J Hepatol. 2017; 67: 370-398.

7. Yuen MF, Sablon E, Hui CK et al. Factors associated with hepatitis B virus DNA breakthrough in patients receiving prolonged lamivudine therapy. Hepatology 2001; 34: 785-791. 
8. Zhou R, Pan F, Lin C et al. Baseline prognostic factors and statistic model to predict early virological response in lamivudine-treated patients with chronic hepatitis B. Int. J Clin. Exp. Med. 2015; 8(10): 19258-19265

9. Wang GL, Liu Y, Qui P et al. Cost-effectiveness of lamivudine, telbivudine, adefovir dipivoxil and entecavir on decompensated hepatitis B virus-related cirrhosis. Eur. Rev. Med. Pharmacol. Sci. 2016; 20: 866-872.

10. Lok ASF, Lai C-L, Leung N, et al. Long-term safety of lamivudine treatment in patients with chronic hepatitis B. Gastroenterology 2003;125:1714-1722

11. Wright TL. Clinical trial results and treatment resistance with lamivudine in hepatitis B. Sem. Liv. Dis. 2004; 24 (Suppl. 1): 31-38.

\section{Corresponding author:}

Deian Jelev,

Clinic of Gastroenterology,

St. Ivan Rilsky University Hospital

Sofia Medical University

15, Acad. Ivan Geshov blvd

1431 Sofia, Bulgaria

email: detjelev@gmail.com 